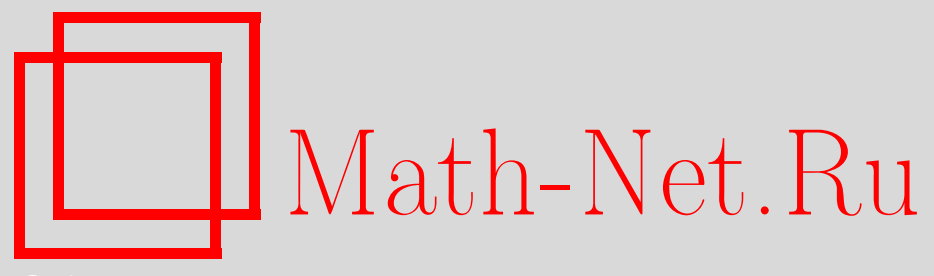

О. И. Мохов, Теория подмногообразий, уравнения ассоциативности двумерных топологических квантовых теорий поля и фробениусовы многообразия, ТМФ, 2007, том 152, номер 2, 368-376

DOI: https://doi.org/10.4213/tmf6093

Использование Общероссийского математического портала Math-Net.Ru подразумевает, что вы прочитали и согласны с пользовательским соглашением http://www . mathnet.ru/rus/agreement

Параметры загрузки:

IP: 3.82 .47 .9

26 апреля 2023 г., $14: 15: 34$

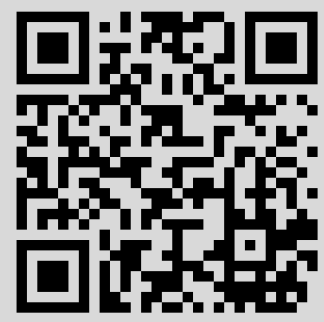




\title{
ТЕОРИЯ ПОДМНОГООБРАЗИЙ, УРАВНЕНИЯ АССОЦИАТИВНОСТИ ДВУМЕРНЫХ ТОПОЛОГИЧЕСКИХ КВАНТОВЫХ ТЕОРИЙ ПОЛЯ И ФРОБЕНИУСОВЫ МНОГООБРАЗИЯ
}

\begin{abstract}
Доказано, что уравнения ассоциативности двумерных топологических квантовых теорий поля являются естественными редукциями фундаментальных нелинейных уравнений теории подмногообразий в псевдоевклидовых пространствах и задают естественный класс потенциальных плоских подмногообразий без кручения. Показано, что все потенциальные плоские подмногообразия без кручения в псевдоевклидовых пространствах имеют естественные структуры фробениусовых алгебр на своих касательных пространствах. Эти фробениусовы структуры порождаются соответствующими плоской первой квадратичной формой и множеством вторых квадратичных форм подмногообразий (фактически структурные константы задаются множеством операторов Вайнгартена подмногообразий). Доказано, что локально каждое $N$-мерное фробениусово многообразие может быть представлено как потенциальное плоское подмногообразие без кручения в $2 N$-мерном псевдоевклидовом пространстве. Нашей конструкцией это подмногообразие определено однозначно с точностью до движений. Более того, рассмотрена нелинейная система, которая является естественным обобщением уравнений ассоциативности, а именно система, описывающая все плоские подмногообразия без кручения в псевдоевклидовых пространствах, и доказано, что эта система является интегрируемой методом обратной задачи рассеяния.
\end{abstract}

Ключевые слова: фробениусово многообразие, подмногообразие псевдоевклидова пространства, плоское подмногообразие, подмногообразие с плоской нормальной связностью, плоское подмногообразие без кручения, уравнения ассоциативности двумерных топологических квантовых теорий поля, интегрируемая система.

${ }^{*}$ Институт теоретической физики им. Л.Д. Ландау РАН, Москва, Россия; Московский государственный университет, Москва, Россия. E-mail: mokhov@mi.ras.ru; mokhov@landau.ac.ru; mokhov@bk.ru 
Памяти моей замечательной мамы Майи Николаевнъ Моховой

$(4.05 .1926-12.09 .2006)$

\section{1. ВВЕДЕНИЕ. УРАВНЕНИЯ АССОЦИАТИВНОСТИ И ФРОБЕНИУСОВЫ СТРУКТУРЫ}

В данной статье мы доказываем, что уравнения ассоциативности двумерных топологических квантовых теорий поля (уравнения Виттена-Дейкграфа-ВерлиндеВерлинде и Дубровина, см. работу [1]) для функции (потенииала или предпотеницала) $\Phi=\Phi\left(u^{1}, \ldots, u^{N}\right)$,

$$
\sum_{k=1}^{N} \sum_{l=1}^{N} \frac{\partial^{3} \Phi}{\partial u^{i} \partial u^{j} \partial u^{k}} \eta^{k l} \frac{\partial^{3} \Phi}{\partial u^{l} \partial u^{m} \partial u^{n}}=\sum_{k=1}^{N} \sum_{l=1}^{N} \frac{\partial^{3} \Phi}{\partial u^{i} \partial u^{m} \partial u^{k}} \eta^{k l} \frac{\partial^{3} \Phi}{\partial u^{l} \partial u^{j} \partial u^{n}},
$$

где $\eta^{i j}$ - произвольная постоянная невырожденная симметричная матрица, $\eta^{i j}=\eta^{j i}$, $\eta^{i j}=$ const $\operatorname{det}\left(\eta^{i j}\right) \neq 0$, являются очень естественными редукциями фундаментальных нелинейных уравнений теории подмногообразий в псевдоевклидовых пространствах и задают естественный класс потенциальных плоских подмногообразий без кручения. Все потенциальные плоские подмногообразия без кручения в псевдоевклидовых пространствах имеют естественные структуры фробениусовых алгебр на своих касательных пространствах. Эти фробениусовы структуры порождаются соответствующими плоской первой квадратичной формой и множеством вторых квадратичных форм подмногообразий (фактически структурные константы задаются множеством операторов Вайнгартена подмногообразий). Напомним, что каждое решение $\Phi\left(u^{1}, \ldots, u^{N}\right)$ уравнений ассоциативности (1.1) задает $N$-параметрические деформации фробениусовых алгебр, т.е. коммутативных ассоциативных алгебр с заданными на них невырожденными инвариантными симметричными билинейными формами. Действительно, рассмотрим алгебры $A(u)$ в $N$-мерном векторном пространстве с базисом $e_{1}, \ldots, e_{N}$ и умножением (см. работу [1])

$$
e_{i} \circ e_{j}=c_{i j}^{k}(u) e_{k}, \quad c_{i j}^{k}(u)=\eta^{k s} \frac{\partial^{3} \Phi}{\partial u^{s} \partial u^{i} \partial u^{j}} e_{k} .
$$

Для всех значений параметров $u=\left(u^{1}, \ldots, u^{N}\right)$ алгебры $A(u)$ коммутативны, $e_{i} \circ e_{j}=$ $e_{j} \circ e_{i}$, а условие ассоциативности

$$
\left(e_{i} \circ e_{j}\right) \circ e_{k}=e_{i} \circ\left(e_{j} \circ e_{k}\right)
$$

в алгебрах $A(u)$ эквивалентно уравнениям (1.1). Матрица $\eta_{i j}$, обратная матрице $\eta^{i j}$, $\eta^{i s} \eta_{s j}=\delta_{j}^{i}$, определяет невырожденную инвариантную симметричную билинейную форму на алгебрах $A(u)$,

$$
\left\langle e_{i}, e_{j}\right\rangle=\eta_{i j}, \quad\left\langle e_{i} \circ e_{j}, e_{k}\right\rangle=\left\langle e_{i}, e_{j} \circ e_{k}\right\rangle .
$$

Напомним, что локально на любом фробениусовом многообразии (см. работу [1]) на касательном пространстве в каждой точке имеется структура фробениусовой алгебры (1.2)-(1.4), которая определяется некоторым решением уравнений ассоциативности (1.1) и гладко зависит от точки. Кроме того, на фробениусовых многообразиях

6 Теоретическая и математическая физика, т. 152, № 2, 2007 г. 
требуется еще выполнение некоторых дополнительных условий, которые мы здесь не рассматриваем. В данной статье мы доказываем, что каждое $N$-мерное фробениусово многообразие локально может быть представлено как потенциальное плоское подмногообразие без кручения в $2 N$-мерном псевдоевклидовом пространстве. Нашей конструкцией это подмногообразие определено однозначно с точностью до движений. Более того, мы рассматриваем нелинейную систему, которая является естественным обобщением уравнений ассоциативности (1.1), а именно систему, описывающую все плоские подмногообразия без кручения в псевдоевклидовых пространствах, и доказываем, что эта система является интегрируемой методом обратной задачи рассеяния. Связь нашей конструкции с интегрируемыми иерархиями, нелокальными гамильтоновыми операторами гидродинамического типа с плоскими метриками, пучками скобок Пуассона и операторами рекурсии может быть найдена в работе автора [2]. Результаты приложений этой конструкции в теории фробениусовых многообразий будут опубликованы в отдельной статье.

\section{2. ФУНДАМЕНТАЛЬНЫЕ НЕЛИНЕЙНЫЕ УРАВНЕНИЯ ТЕОРИИ ПОДМНОГООБРАЗИЙ В ЕВКЛИДОВЫХ ПРОСТРАНСТВАХ}

Рассмотрим произвольное гладкое $N$-мерное подмногообразие $M^{N}$ в $(N+L)$-мерном евклидовом пространстве $E^{N+L}, M^{N} \subset E^{N+L}$, и введем стандартные классические обозначения. Пусть подмногообразие $M^{N}$ локально задано некоторой гладкой вектор-функцией $r\left(u^{1}, \ldots, u^{N}\right)$, зависящей от $N$ независимых переменных $\left(u^{1}, \ldots, u^{N}\right)$ (некоторые независимые параметры на подмногообразии), $r\left(u^{1}, \ldots, u^{N}\right)=\left(z^{1}\left(u^{1}, \ldots, u^{N}\right), \ldots, z^{N+L}\left(u^{1}, \ldots, u^{N}\right)\right)$, где $\left(z^{1}, \ldots, z^{N+L}\right)-$ координаты в евклидовом пространстве $E^{N+L},\left(z^{1}, \ldots, z^{N+L}\right) \in E^{N+L},\left(u^{1}, \ldots, u^{N}\right)$ - локальные координаты (параметры) на $M^{N}, \operatorname{rank}\left(\partial z^{i} / \partial u^{j}\right)=N$ (здесь $1 \leqslant i \leqslant N+L$, $1 \leqslant j \leqslant N)$. Тогда $\partial r / \partial u^{i}=r_{u^{i}}, 1 \leqslant i \leqslant N,-$ касательные векторы в произвольной точке $u=\left(u^{1}, \ldots, u^{N}\right)$ на $M^{N}$. Пусть $\mathbf{N}_{u}-$ нормальное пространство подмногообразия $M^{N}$ в произвольной точке $u=\left(u^{1}, \ldots, u^{N}\right)$ на $M^{N}, N_{u}=\left\langle n_{1}, \ldots, n_{L}\right\rangle$, где $n_{\alpha}$, $1 \leqslant \alpha \leqslant L,-$ ортонормированный базис нормального пространства (ортонормированные нормали), $\left(n_{\alpha}, r_{u^{i}}\right)=0,1 \leqslant \alpha \leqslant L, 1 \leqslant i \leqslant N,\left(n_{\alpha}, n_{\beta}\right)=0,1 \leqslant \alpha, \beta \leqslant L$, $\alpha \neq \beta$, и $\left(n_{\alpha}, n_{\alpha}\right)=1,1 \leqslant \alpha \leqslant L$.

Тогда $\mathbf{I}=d s^{2}=g_{i j}(u) d u^{i} d u^{j}, g_{i j}(u)=\left(r_{u^{i}}, r_{u^{j}}\right)$, - первая квадратичная форма и $\mathbf{I I}_{\alpha}=\omega_{\alpha, i j}(u) d u^{i} d u^{j}, \omega_{\alpha, i j}(u)=\left(n_{\alpha}, r_{u^{i} u^{j}}\right), 1 \leqslant \alpha \leqslant L,-$ вторые квадратичные формы подмногообразия $M^{N}$.

Поскольку множество векторов $\left(r_{u^{1}}(u), \ldots, r_{u^{N}}(u), n_{1}(u), \ldots, n_{L}(u)\right)$ образует базис в $E^{N+L}$ в каждой точке подмногообразия $M^{N}$, мы можем разложить каждый из векторов $n_{\alpha, u^{i}}(u), 1 \leqslant \alpha \leqslant L, 1 \leqslant i \leqslant N$, по этому базису, а именно $n_{\alpha, u^{i}}(u)=A_{\alpha, i}^{k}(u) r_{u^{k}}(u)+\varkappa_{\alpha \beta, i}(u) n_{\beta}(u)$, где $A_{\alpha, i}^{k}(u)$ и $\varkappa_{\alpha \beta, i}(u)-$ некоторые коэффициенты, зависящие от $u$ (разложение Вайнгартена). Легко доказать, что $A_{\alpha, i}^{k}(u)=-\omega_{\alpha, i j}(u) g^{j k}(u)$, где $g^{j k}(u)$ - контравариантная метрика, обратная первой квадратичной форме $g_{i j}(u), g^{i s}(u) g_{s j}(u)=\delta_{j}^{i}$. Коэффициенты $\varkappa_{\alpha \beta, i}(u)$ называются коэффициентами кручения подмногообразия $M^{N}, \varkappa_{\alpha \beta, i}(u)=\left(n_{\alpha, u^{i}}(u), n_{\beta}(u)\right)$. Легко также доказать, что коэффициенты $\varkappa_{\alpha \beta, i}(u)$ кососимметричны по индексам $\alpha$ 
и $\beta, \varkappa_{\alpha \beta, i}(u)=-\varkappa_{\beta \alpha, i}(u)$, и образуют ковариантные тензоры (1-формы) по индексу $i$ на подмногообразии $M^{N}$. 1-Формы $\varkappa_{\alpha \beta, i}(u) d u^{i}$ называются формами кручения подмногообразия $M^{N}$.

Хорошо известно, что для каждого подмногообразия $M^{N}$ формы $g_{i j}(u), \omega_{\alpha, i j}(u)$ и $\varkappa_{\alpha \beta, i}(u)$ удовлетворяют уравнениям Гаусса, уравнениям Кодацци и уравнениям Риччи, которые являются фундаментальными уравнениями теории подмногообразий. В нашем случае уравнения Гаусса имеют вид

$$
R_{i j k l}(u)=\sum_{\alpha=1}^{L}\left(\omega_{\alpha, j l}(u) \omega_{\alpha, i k}(u)-\omega_{\alpha, j k}(u) \omega_{\alpha, i l}(u)\right),
$$

где $R_{i j k l}(u)$ - тензор римановой кривизны первой квадратичной формы $g_{i j}(u)$; уравнения Кодацци имеют вид

$$
\nabla_{k}\left(\omega_{\alpha, i j}(u)\right)-\nabla_{j}\left(\omega_{\alpha, i k}(u)\right)=\varkappa_{\alpha \beta, k}(u) \omega_{\beta, i j}(u)-\varkappa_{\alpha \beta, j}(u) \omega_{\beta, i k}(u),
$$

где $\nabla_{k}$ - оператор ковариантного дифференцирования, порождаемый связностью Леви-Чивита первой квадратичной формы $g_{i j}(u)$; уравнения Риччи имеют вид

$$
\begin{gathered}
\nabla_{k}\left(\varkappa_{\alpha \beta, i}(u)\right)-\nabla_{i}\left(\varkappa_{\alpha \beta, k}(u)\right)+\sum_{\gamma=1}^{L}\left(\varkappa_{\alpha \gamma, i}(u) \varkappa_{\gamma \beta, k}(u)-\varkappa_{\alpha \gamma, k}(u) \varkappa_{\gamma \beta, i}(u)\right)+ \\
+\left(\omega_{\alpha, k l}(u) \omega_{\beta, j i}(u)-\omega_{\alpha, i l}(u) \omega_{\beta, j k}(u)\right) g^{l j}(u)=0 .
\end{gathered}
$$

Теорема 2.1 (Бонне). Пусть $K^{N}$ - произволъное гладкое $N$-мерное риманово многообразие с метрикой $g_{i j}(u) d u^{i} d u^{j}$. Пусть в односвязной области многообразия $K^{N}$ заданъ некоторье 2-формы $\omega_{\alpha, i j}(u) d u^{i} d u^{j}, 1 \leqslant \alpha \leqslant L$, и некоторые $1-\oint о р м и ~ \varkappa_{\alpha \beta, i}(u), 1 \leqslant \alpha, \beta \leqslant L . E с л u \omega_{\alpha, i j}(u)=\omega_{\alpha, j i}(u), \varkappa_{\alpha \beta, i}(u)=-\varkappa_{\beta \alpha, i}(u) u \partial л я$ борм $g_{i j}(u), \omega_{\alpha, i j}(u)$ и $\varkappa_{\alpha \beta, i}(u)$ выполняются уравнения Гаусса (2.1), уравнения Кодачии (2.2) и уравнения Риччи (2.3), то существует единственное (с точностью до движений) гладкое $N$-мерное подмногообразие $M^{N}$ в $(N+L)$-мерном евклидовом пространстве $E^{N+L}$ с первой квадратичной формой $d s^{2}=g_{i j}(u) d u^{i} d u^{j}$, вторьми квадратичными формами $\omega_{\alpha, i j}(u) d u^{i} d u^{j}$ и формами кручения $\varkappa_{\alpha \beta, i}(u) d u^{i}$.

Полностью аналогичные фундаментальные уравнения и теорема Бонне справедливы для всех вполне неизотропных подмногообразий в псевдоевклидовых пространcmваx (напомним, что для подмногообразия произвольного псевдоевклидова пространства $E_{n}^{m}$ метрика, индуцированная на подмногообразии из объемлющего псевдоевклидова пространства $E_{n}^{m}$, является невырожденной тогда и только тогда, когда это подмногообразие вполне неизотропно, т.е. ни в одной из своих точек не касается изотропных конусов объемлющего псевдоевклидова пространства $\left.E_{n}^{m}\right)$.

\section{3. ПЛОСКИЕ ПОДМНОГООБРАЗИЯ БЕЗ КРУЧЕНИЯ В ПСЕВДОЕВКЛИДОВЫХ ПРОСТРАНСТВАХ}

Рассмотрим вполне неизотропные гладкие $N$-мерные подмногообразия без кручения в $(N+L)$-мерном псевдоевклидовом пространстве, т.е. все формы кручения подмногообразий рассматриваемого класса тождественно равны нулю: $\varkappa_{\alpha \beta, i}(u)=0$. 
Мы также будем использовать в нормальных пространствах $N_{u}$ базисы $n_{\alpha}, 1 \leqslant$ $\alpha \leqslant L$, с произвольными допустимыми матрицами Грама $\mu_{\alpha \beta},\left(n_{\alpha}, n_{\beta}\right)=\mu_{\alpha \beta}$, $\mu_{\alpha \beta}=$ const, $\mu_{\alpha \beta}=\mu_{\beta \alpha}$, $\operatorname{det} \mu_{\alpha \beta} \neq 0$ (сигнатура метрики $\mu_{\alpha \beta}$ определяется сигнатурой первой квадратичной формы подмногообразия и сигнатурой объемлющего псевдоевклидова пространства).

Для $N$-мерных подмногообразий без кручения в $(N+L)$-мерном псевдоевклидовом пространстве мы получаем следующую систему фундаментальных уравнений: уравнения Гаусса

$$
R_{i j k l}(u)=\sum_{\alpha=1}^{L} \sum_{\beta=1}^{L} \mu^{\alpha \beta}\left(\omega_{\alpha, i k}(u) \omega_{\beta, j l}(u)-\omega_{\alpha, i l}(u) \omega_{\beta, j k}(u)\right),
$$

где $\mu^{\alpha \beta}$ - матрица, обратная матрице $\mu_{\alpha \beta}, \mu^{\alpha \gamma} \mu_{\gamma \beta}=\delta_{\beta}^{\alpha}$; уравнения Кодацци

$$
\nabla_{k}\left(\omega_{\alpha, i j}(u)\right)=\nabla_{j}\left(\omega_{\alpha, i k}(u)\right)
$$

и уравнения Риччи

$$
g^{i j}(u)\left(\omega_{\alpha, i k}(u) \omega_{\beta, j l}(u)-\omega_{\alpha, i l}(u) \omega_{\beta, j k}(u)\right)=0 .
$$

Пусть теперь $g_{i j}(u)$ - плоская метрика, т.е. мы рассматриваем плоские $N$-мерные подмногообразия $M^{N}$ без кручения в $(N+L)$-мерном псевдоевклидовом пространстве. Тогда мы можем считать, что $u=\left(u^{1}, \ldots, u^{N}\right)$ - некоторые плоские координаты метрики $g_{i j}(u)$ на $M^{N}$. В плоских координатах метрика является постоянной невырожденной симметричной матрицей $\eta_{i j}, \eta_{i j}=\eta_{j i}, \eta_{i j}=\mathrm{const}, \operatorname{det}\left(\eta_{i j}\right) \neq 0$, a уравнения Кодацци (3.2) принимают вид

$$
\frac{\partial \omega_{\alpha, i j}}{\partial u^{k}}=\frac{\partial \omega_{\alpha, i k}}{\partial u^{j}}
$$

Таким образом, локально существуют некоторые функции $\chi_{\alpha, i}(u), 1 \leqslant \alpha \leqslant L, 1 \leqslant$ $i \leqslant N$, такие, что

$$
\omega_{\alpha, i j}(u)=\frac{\partial \chi_{\alpha, i}}{\partial u^{j}}
$$

Из симметрии вторых квадратичных форм $\omega_{\alpha, i j}(u)=\omega_{\alpha, j i}(u)$ мы получаем

$$
\frac{\partial \chi_{\alpha, i}}{\partial u^{j}}=\frac{\partial \chi_{\alpha, j}}{\partial u^{i}}
$$

Следовательно, локально существуют некоторые функции $\psi_{\alpha}(u), 1 \leqslant \alpha \leqslant L$, такие, что

$$
\chi_{\alpha, i}(u)=\frac{\partial \psi_{\alpha}}{\partial u^{i}}, \quad \omega_{\alpha, i j}(u)=\frac{\partial^{2} \psi_{\alpha}}{\partial u^{i} \partial u^{j}} .
$$

Таким образом, мы доказали следующую важную лемму.

ЛЕмма. Все вторые квадратичные формы каждого плоского подмногообразия без кручения в псевдоевклидовом пространстве являются гессианами в любых плоских координатах в любой односвязной области на подмногообразии. 
Более того, в любых плоских координатах уравнения Гаусса (3.1) имеют вид

$$
\sum_{\alpha=1}^{L} \sum_{\beta=1}^{L} \mu^{\alpha \beta}\left(\frac{\partial^{2} \psi_{\alpha}}{\partial u^{i} \partial u^{k}} \frac{\partial^{2} \psi_{\beta}}{\partial u^{j} \partial u^{l}}-\frac{\partial^{2} \psi_{\alpha}}{\partial u^{i} \partial u^{l}} \frac{\partial^{2} \psi_{\beta}}{\partial u^{j} \partial u^{k}}\right)=0,
$$

а уравнения Риччи (3.3) -

$$
\sum_{i=1}^{N} \sum_{j=1}^{N} \eta^{i j}\left(\frac{\partial^{2} \psi_{\alpha}}{\partial u^{i} \partial u^{k}} \frac{\partial^{2} \psi_{\beta}}{\partial u^{j} \partial u^{l}}-\frac{\partial^{2} \psi_{\alpha}}{\partial u^{i} \partial u^{l}} \frac{\partial^{2} \psi_{\beta}}{\partial u^{j} \partial u^{k}}\right)=0
$$

где $\eta^{i j}$ - матрица, обратная матрице $\eta_{i j}, \eta^{i s} \eta_{s j}=\delta_{j}^{i}$.

Теорема 3.1. Класс $N$-мерных плоских подмногообразий без кручения в $(N+L)$-мерных псевдоевклидовых пространствах описывается (в плоских координатах) системой нелинейных уравнений (3.8), (3.9) для функций $\psi_{\alpha}(u), 1 \leqslant \alpha \leqslant L$. Здесъ $\eta^{i j}$ и $\mu^{\alpha \beta}$ - произвольные постоянные невырожденные симметричные матриu, $b, \eta^{i j}=\eta^{j i}, \eta^{i j}=\mathrm{const}, \operatorname{det}\left(\eta^{i j}\right) \neq 0, \mu^{\alpha \beta}=\mathrm{const}, \mu^{\alpha \beta}=\mu^{\beta \alpha}, \operatorname{det} \mu^{\alpha \beta} \neq 0$, сигнатура обгемлющего $(N+L)$-мерного псевдоевклидова пространства является суммой сигнатур метрик $\eta^{i j} u \mu^{\alpha \beta}, \mathbf{I}=d s^{2}=\eta_{i j} d u^{i} d u^{j}$ - первая квадратичная форма, где $\eta_{i j}$ - матриц, обратная матрище $\eta^{i j}, \eta^{i s} \eta_{s j}=\delta_{j}^{i}, \mathbf{I I}_{\alpha}=\left(\partial^{2} \psi_{\alpha} / \partial u^{i} \partial u^{j}\right) d u^{i} d u^{j}$, $1 \leqslant \alpha \leqslant L,-$ вторые квадратичные формы, задаваемые гессианами функций $\psi_{\alpha}(u)$, $1 \leqslant \alpha \leqslant L$.

В соответствии с теоремой Бонне любое решение $\psi_{\alpha}(u), 1 \leqslant \alpha \leqslant L$, нелинейной системы (3.8), (3.9) определяет единственное (с точностью до движений) $N$-мерное плоское подмногообразие без кручения в соответствующем $(N+L)$-мерном псевдоевклидовом пространстве с первой квадратичной формой $\eta_{i j} d u^{i} d u^{j}$ и вторыми квадратичными формами $\omega_{\alpha}(u)=\left(\partial^{2} \psi_{\alpha} / \partial u^{i} \partial u^{j}\right) d u^{i} d u^{j}, 1 \leqslant \alpha \leqslant L$, задаваемыми гессианами функций $\psi_{\alpha}(u), 1 \leqslant \alpha \leqslant L$. Очевидно, что мы всегда можем добавить произвольные слагаемые, линейные по координатам $\left(u^{1}, \ldots, u^{N}\right)$, к любому решению системы (3.8), (3.9), но множество вторых квадратичных форм и соответствующее подмногообразие останутся теми же самыми. Более того, любые два множества вторых квадратичных форм вида $\omega_{\alpha, i j}(u)=\partial^{2} \psi_{\alpha} / \partial u^{i} \partial u^{j}, 1 \leqslant \alpha \leqslant L$, совпадают тогда и только тогда, когда соответствующие функции $\psi_{\alpha}(u), 1 \leqslant \alpha \leqslant L$, совпадают с точностью до линейных по координатам слагаемых, поэтому мы здесь не должны различать решения нелинейной системы (3.8), (3.9), отличающиеся на слагаемые, линейные по координатам $\left(u^{1}, \ldots, u^{N}\right)$.

Теорема 3.2. Нелинейная система (3.8), (3.9) является интегрируемой методом обратной задачи рассеяния.

Рассмотрим следующую линейную задачу для вектор-функций $\partial a(u) / \partial u^{i}$ и $b_{\alpha}(u)$, $1 \leqslant \alpha \leqslant L:$

$$
\frac{\partial^{2} a}{\partial u^{i} \partial u^{j}}=\lambda \mu^{\alpha \beta} \omega_{\alpha, i j}(u) b_{\beta}(u), \quad \frac{\partial b_{\alpha}}{\partial u^{i}}=\rho \eta^{k j} \omega_{\alpha, i j}(u) \frac{\partial a}{\partial u^{k}},
$$

где $\eta^{i j}, 1 \leqslant i, j \leqslant N$, и $\mu^{\alpha \beta}, 1 \leqslant \alpha, \beta \leqslant L,-$ произвольные постоянные невырожденные симметричные матрицы, $\eta^{i j}=\eta^{j i}, \eta^{i j}=\mathrm{const}, \operatorname{det}\left(\eta^{i j}\right) \neq 0, \mu^{\alpha \beta}=\mathrm{const}$, 
$\mu^{\alpha \beta}=\mu^{\beta \alpha}, \operatorname{det} \mu^{\alpha \beta} \neq 0$; очевидно, что при этом коэффициенты $\omega_{\alpha, i j}(u), 1 \leqslant \alpha \leqslant L$, должны быть симметричными матричными функциями, $\omega_{\alpha, i j}(u)=\omega_{\alpha, j i}(u) ; \lambda$ и $\rho-$ произвольные константы (параметры).

Условия совместности для линейной системы (3.10) эквивалентны нелинейной системе (3.8), (3.9), описывающей класс $N$-мерных плоских подмногообразий без кручения в $(N+L)$-мерных псевдоевклидовых пространствах. Действительно, мы имеем

$$
\begin{aligned}
\frac{\partial^{3} a}{\partial u^{i} \partial u^{j} \partial u^{k}} & =\lambda \mu^{\alpha \beta} \frac{\partial \omega_{\alpha, i j}}{\partial u^{k}} b_{\beta}(u)+\lambda \mu^{\alpha \beta} \omega_{\alpha, i j}(u) \frac{\partial b_{\beta}}{\partial u^{k}}= \\
& =\lambda \mu^{\alpha \beta} \frac{\partial \omega_{\alpha, i j}}{\partial u^{k}} b_{\beta}(u)+\lambda \mu^{\alpha \beta} \omega_{\alpha, i j}(u) \rho \eta^{l s} \omega_{\beta, k s}(u) \frac{\partial a}{\partial u^{l}}= \\
& =\lambda \mu^{\alpha \beta} \frac{\partial \omega_{\alpha, i k}}{\partial u^{j}} b_{\beta}(u)+\lambda \mu^{\alpha \beta} \omega_{\alpha, i k}(u) \rho \eta^{l s} \omega_{\beta, j s}(u) \frac{\partial a}{\partial u^{l}}
\end{aligned}
$$

откуда получаем

$$
\frac{\partial \omega_{\alpha, i j}(u)}{\partial u^{k}}=\frac{\partial \omega_{\alpha, i k}(u)}{\partial u^{j}}
$$

и

$$
\mu^{\alpha \beta} \omega_{\alpha, i j}(u) \omega_{\beta, k s}(u)=\mu^{\alpha \beta} \omega_{\alpha, i k}(u) \omega_{\beta, j s}(u) .
$$

Кроме того,

$$
\begin{aligned}
\frac{\partial^{2} b_{\alpha}}{\partial u^{i} \partial u^{l}} & =\rho \eta^{k j} \frac{\partial \omega_{\alpha, i j}}{\partial u^{l}} \frac{\partial a}{\partial u^{k}}+\rho \eta^{k j} \omega_{\alpha, i j}(u) \frac{\partial^{2} a}{\partial u^{k} \partial u^{l}}= \\
& =\rho \eta^{k j} \frac{\partial \omega_{\alpha, i j}}{\partial u^{l}} \frac{\partial a}{\partial u^{k}}+\rho \eta^{k j} \omega_{\alpha, i j}(u) \lambda \mu^{\gamma \beta} \omega_{\gamma, k l}(u) b_{\beta}(u)= \\
& =\rho \eta^{k j} \frac{\partial \omega_{\alpha, l j}}{\partial u^{i}} \frac{\partial a}{\partial u^{k}}+\rho \eta^{k j} \omega_{\alpha, l j}(u) \lambda \mu^{\gamma \beta} \omega_{\gamma, k i}(u) b_{\beta}(u),
\end{aligned}
$$

откуда имеем

$$
\frac{\partial \omega_{\alpha, i j}}{\partial u^{l}}=\frac{\partial \omega_{\alpha, l j}}{\partial u^{i}}
$$

и

$$
\eta^{k j} \omega_{\alpha, i j}(u) \omega_{\gamma, k l}(u)=\eta^{k j} \omega_{\alpha, l j}(u) \omega_{\gamma, k i}(u)
$$

Из (3.12) и (3.15) следует, что локально существуют некоторые функции $\psi_{\alpha}(u)$, $1 \leqslant \alpha \leqslant L$, такие, что

$$
\omega_{\alpha, i j}(u)=\frac{\partial^{2} \psi_{\alpha}}{\partial u^{i} \partial u^{j}}
$$

тогда соотношения (3.13) и (3.16) эквивалентны нелинейной системе (3.8), (3.9) для функций $\psi_{\alpha}(u), 1 \leqslant \alpha \leqslant L$.

В произвольных локальных координатах мы получаем следующее интегрируемое описание всех $N$-мерных плоских подмногообразий без кручения в $(N+L)$-мерных псевдоевклидовых пространствах.

Теорема 3.3. Для каждого $N$-мерного плоского подмногообразия без кручения в $(N+L)$-мерном псевдоевклидовом пространстве с плоской первой квадратичной формой $g_{i j}(u)$ локально существуют функиии $\psi_{\alpha}(u), 1 \leqslant \alpha \leqslant L$, такие, что вторые квадратичные формы имеют вид

$$
\left(w_{\alpha}\right)_{i j}(u)=\nabla_{i} \nabla_{j} \psi_{\alpha},
$$


где $\nabla_{i}$ - оператор ковариантного дифферениирования, определяемый связностъю Леви-Чивита, порождаемой метрикой $g_{i j}(u)$. Класс $N$-мерных плоских подмногообразий без кручения в $(N+L)$-мерных псевдоевклидовых пространствах описывается следующей интегрируемой системой нелинейных уравнений для функиий $\psi_{\alpha}(u), 1 \leqslant \alpha \leqslant L$ :

$$
\begin{aligned}
\sum_{n=1}^{N} \nabla^{n} \nabla_{i} \psi_{\alpha} \nabla_{n} \nabla_{l} \psi_{\beta} & =\sum_{n=1}^{N} \nabla^{n} \nabla_{i} \psi_{\beta} \nabla_{n} \nabla_{l} \psi_{\alpha} \\
\sum_{\alpha=1}^{L} \sum_{\beta=1}^{L} \mu^{\alpha \beta} \nabla_{i} \nabla_{j} \psi_{\alpha} \nabla_{k} \nabla_{l} \psi_{\beta} & =\sum_{\alpha=1}^{L} \sum_{\beta=1}^{L} \mu^{\alpha \beta} \nabla_{i} \nabla_{k} \psi_{\alpha} \nabla_{j} \nabla_{l} \psi_{\beta},
\end{aligned}
$$

где $\nabla_{i}$ - ковариантное дифференцирование, определяемое связностью Леви- Чивита, порождаемой плоской метрикой $g_{i j}(u), \nabla^{i}=g^{i s}(u) \nabla_{s}, g^{i s}(u) g_{s j}(u)=\delta_{j}^{i}$. Более того, в этом случае системы гидродинамического типа

$$
u_{t_{\alpha}}^{i}=\left(\nabla^{i} \nabla_{j} \psi_{\alpha}\right) u_{x}^{j}, \quad 1 \leqslant \alpha \leqslant L
$$

являются коммутирующими интегрируемыми бигамильтоновыми системами гидродинамического типа.

\section{4. РЕДУКЦИЯ К УРАВНЕНИЯМ АССОЦИАТИВНОСТИ ДВУМЕРНЫХ ТОПОЛОГИЧЕСКИХ КВАНТОВЫХ ТЕОРИЙ ПОЛЯ И ПОТЕНЦИАЛЬНЫМ ПЛОСКИМ ПОДМНОГООБРАЗИЯМ БЕЗ КРУЧЕНИЯ В ПСЕВДОЕВКЛИДОВЫХ ПРОСТРАНСТВАХ}

Теперь мы найдем также некоторые естественные и очень важные интегрируемые редукции нелинейной системы (3.8), (3.9).

Теорема 4.1. Если взять $L=N, \mu^{i j}=c \eta^{i j}, 1 \leqslant i, j \leqslant N$, где $c$ - произвольная ненулевая константа, $u \psi_{\alpha}(u)=\partial \Phi / \partial u^{\alpha}, 1 \leqslant \alpha \leqslant N$, где $\Phi=\Phi\left(u^{1}, \ldots, u^{N}\right)$, то уравнения Гаусса (3.8) совпадут с уравнениями Риччи (3.9), при этом и те, и другие уравнения совпадут с уравнениями ассоциативности двумерных топологических квантовых теорий поля для потенииала $\Phi(u)$ :

$$
\sum_{i=1}^{N} \sum_{j=1}^{N} \eta^{i j}\left(\frac{\partial^{3} \Phi}{\partial u^{i} \partial u^{m} \partial u^{k}} \frac{\partial^{3} \Phi}{\partial u^{j} \partial u^{n} \partial u^{l}}-\frac{\partial^{3} \Phi}{\partial u^{i} \partial u^{m} \partial u^{l}} \frac{\partial^{3} \Phi}{\partial u^{j} \partial u^{n} \partial u^{k}}\right)=0 .
$$

ТеоремА 4.2. Уравнения ассоииативности двумерных топологических квантовых теорий поля описывают специальный класс $N$-мерных плоских подмногообразий без кручения в $2 N$-мерных псевдоевклидовых пространствах (класс потенциальных плоских подмногообразий без кручения).

ОПредЕЛЕНиЕ. Плоское $N$-мерное подмногообразие без кручения в $2 N$-мерном псевдоевклидовом пространстве с плоской первой квадратичной формой $g_{i j}(u) d u^{i} d u^{j}$ называется потенииальным, если локально всегда существует некоторая функция $\Phi(u)$ в окрестности на подмногообразии такая, что локально (в этой 
окрестности) вторые квадратичные формы этого подмногообразия имеют вид

$$
\left(\omega_{i}\right)_{j k}(u) d u^{j} d u^{k}=\left(\nabla_{i} \nabla_{j} \nabla_{k} \Phi(u)\right) d u^{j} d u^{k}, \quad 1 \leqslant i \leqslant N,
$$

где $\nabla_{i}$ - оператор ковариантного дифференцирования, определяемый связностью Леви-Чивита, порождаемой плоской метрикой $g_{i j}(u)$.

По теореме Бонне любое решение $\Phi(u)$ уравнений ассоциативности (4.1) (с соответствующей постоянной метрикой $\eta_{i j}$ ) определяет единственное (с точностью до движений) $N$-мерное потенциальное плоское подмногообразие без кручения в соответствующем $2 N$-мерном псевдоевклидовом пространстве с первой квадратичной формой $\eta_{i j} d u^{i} d u^{j}$ и вторыми квадратичными формами $\omega_{n}(u)=$ $\left(\partial^{3} \Phi /\left(\partial u^{n} \partial u^{i} \partial u^{j}\right)\right) d u^{i} d u^{j}$, задаваемыми третьими производными потенциала $\Phi(u)$. Мы здесь не различаем решения уравнений ассоциативности (4.1), отличающиеся на слагаемые, квадратичные по координатам $u$.

ТЕОРема 4.3. На каждом потенциальном плоском подмногообразии без кручения в псевдоевклидовом пространстве имеется структура фробениусовой алгебры, задаваемая (в плоских координатах) плоской первой квадратичной формой $\eta_{i j} u$ операторами Вайнгартена $\left(A_{s}\right)_{j}^{i}(u)=-\eta^{i k}\left(\omega_{s}\right)_{k j}(u)$ :

$$
\begin{gathered}
\left\langle e_{i}, e_{j}\right\rangle=\eta_{i j}, \quad e_{i} \circ e_{j}=c_{i j}^{k}(u) e_{k}, \quad e_{i}=\frac{\partial}{\partial u^{i}}, \\
c_{i j}^{k}\left(u^{1}, \ldots, u^{N}\right)=-\left(A_{i}\right)_{j}^{k}(u)=\eta^{k s}\left(\omega_{i}\right)_{s j}\left(u^{1}, \ldots, u^{N}\right) .
\end{gathered}
$$

В произвольных локальных координатах эта фробениусова структура имеет вид

$$
\begin{gathered}
\left\langle e_{i}, e_{j}\right\rangle=g_{i j}, \quad e_{i} \circ e_{j}=c_{i j}^{k}(u) e_{k}, \quad e_{i}=\frac{\partial}{\partial u^{i}}, \\
c_{i j}^{k}\left(u^{1}, \ldots, u^{N}\right)=-\left(A_{i}\right)_{j}^{k}(u)=g^{k s}\left(u^{1}, \ldots, u^{N}\right)\left(\omega_{i}\right)_{s j}\left(u^{1}, \ldots, u^{N}\right),
\end{gathered}
$$

где $g^{i j}(u)$ - контравариантная метрика, обратная первой квадратичной форме $g_{i j}(u), g^{i s}(u) g_{s j}(u)=\delta_{j}^{i} ;\left(\omega_{k}\right)_{i j}(u) d u^{i} d u^{j}, 1 \leqslant k \leqslant N$, - вторые квадратичные формus.

Теорема 4.4. Каждое $N$-мерное фробениусово многообразие локалъно может быть представлено как потенииальное плоское $N$-мерное подмногообразие без кручения в $2 N$-мерном псевдоевклидовом пространстве. Это подмногообразие определяется однозначно с точностъю до движений.

Благодарности. Работа выполнена при финансовой поддержке Института математики Макса Планка (Бонн, Германия), Фонда содействия отечественной науке, РФФИ (грант №05-01-00170) и Программы поддержки ведущих научных школ (грант №НШ-4182.2006.1).

\section{Список литературы}

[1] B. Dubrovin, "Geometry of 2D topological field theories", Integrable Systems and Quantum Groups (Montecatini Terme, Italy, 1993), Lect. Notes Math., 1620, eds. M. Francaviglia, S. Greco, Springer, Berlin, 1996, 120-348; hep-th/9407018.

[2] О.И. Мохов, Функи. анализ и его приложс., 40:1 (2006), 14-29; math.DG/0406292. 